\title{
El regadío y su influencia en la regulación local de temperaturas en superficie en la citricultura mediterránea'
}

\section{Irrigation and its influence on the local regulation of surface temperatures in Mediterranean citriculture}

\author{
José Antonio Albaladejo-García² (1), Francisco Alcon³ (i) \\ y José Miguel Martínez-Paz ${ }^{4}$ (1)
}

\begin{abstract}
RESUMEN
El efecto frío del regadío, dada su influencia en la temperatura de la superficie terrestre (TS), es uno de los servicios ecosistémicos de regulación de los ecosistemas agrarios. En la agricultura de regadío de zonas cálidas este servicio juega un papel destacado al amortiguar los extremos de temperatura. El objetivo de este trabajo es cuantificar y determinar los factores que explican el comportamiento de la TS en áreas de regadío, utilizando como caso de estudio el agroecosistema del cultivo de cítricos en regadío del Campo de Cartagena (Murcia, España). En primer lugar, se demuestra la influencia del regadío en la TS de la zona. Posteriormente se explica el valor de la TS en los regadíos mediante un modelo que relaciona la misma con el índice de vegetación, el contenido de agua del cultivo y la lejanía a la costa. Estos resultados ponen de manifiesto la generación del servicio ecosistémico de regulación climática por los cítricos en regadío.
\end{abstract}

Palabras clave: Ecosistema agrario, Teledetección, Vegetación.

\begin{abstract}
The irrigation cooling effect, given its influence on the land surface temperature (TS), is one of the regulating ecosystem services of agrarian ecosystems. In irrigated agriculture of warm areas, this service plays an important role in cushioning extremes of temperature. The objective of this work is to quantify and determine the factors that explain the behavior of TS in irrigated areas, using as a case study the agroecosystem of citrus cultivation in irrigation of Campo de Cartagena (Murcia, Spain). First, it demonstrates the influence of irrigation on the TS of the area. Subsequently, the value of the TS in the irrigation is explained by a model that relates the same to the vegetation index, the water content of the crop and the distance to the coast. These results show the generation of ecosystem service of climate regulation by citrus in irrigation.
\end{abstract}

Keywords: Agrarian ecosystem, Teledetection, Vegetation.

Una versión preliminar de este trabajo ha sido presentada en las IV Jornadas Doctorales de la Universidad de Murcia (Albaladejo-García et al., 2019)

Departamento de Economía Aplicada. Facultad de Economía y Empresa. Universidad de Murcia. Correo electrónico: joseantonio.albaladejo@um.es Departamento de Economía de la Empresa. Escuela Técnica Superior de Ingeniería Agronómica. Universidad Politécnica de Cartagena. Correo electrónico: francisco.alcon@upct.es

Departamento de Economía Aplicada. Facultad de Economía y Empresa. Universidad de Murcia. Correo electrónico: jmpaz@um.es 
La temperatura de la superficie terrestre (TS), además de ser un factor determinante en el estudio del clima, es un buen indicador del balance energético en la superficie terrestre que puede alterar o modificar la temperatura del aire. La TS, se obtiene de imágenes satélites y permite estudiar fenómenos climáticos a escala local, como son la isla de calor urbana (Estoque et al., 2017; Li et al., 2017; Sun \& Chen, 2017) o el efecto frío de la vegetación (Duveiller et al., 2018; Xu et al., 2017; Zardo et al., 2017) dado que puede verse afectada por los cambios de uso del suelo (Hereher, 2017; Yuan et al., 2017).

El efecto frío de la vegetación es un fenómeno que contribuye al control de la temperatura, básicamente disminuyendo la intensidad de los valores extremos. Esta regulación de temperatura está generada por el efecto oasis de la evaporación del agua y de la fotosíntesis que libera humedad al ambiente. Este efecto es un servicio ecosistémico (SE) de regulación climática (MEA, 2005), o de "regulación local y regional del clima" si se atiende al sistema de clasificación Common International Clasification of Ecosystem Services (CICES). En definitiva, los ecosistemas tienen capacidad para modificar la temperatura (Haines-Young \& Potschin, 2012) y con ello contribuyen al bienestar social, especialmente en climas cálidos.

Este SE ha sido mayormente estudiado a nivel global (Cook et al., 2011; Thiery et al., 2017; Thiery et al., 2020) o regional (Kang \& Eltahir, 2019; Lobell et al., 2008; Guimberteau et al., 2012) a partir de modelos climáticos. Sin embargo, los modelos climáticos pierden precisión en la modelización de los efectos locales debido a su resolución espacial. Por ello, para abordar el estudio de la TS en ecosistemas agrarios concretos hay que recurrir a técnicas en teledetección, obteniendo esta variable de una imagen satélite específica. La teledetección puede facilitar el seguimiento de variables ambientales, como la TS, con una gran resolución espacial (Ej.: Satélites Landsat y Sentinel) o temporal (Ej.: Satélites MODIS y NOAA) (Sánchez-Díaz, 2018). En este sentido cabe destacar el trabajo de Chen et al. (2018) que estudia el papel del maíz en regadío para reducir la temperatura hasta $1^{\circ} \mathrm{C}$ en Nebraska (EE. UU.); el de Wang et al. (2017) sobre el enfriamiento de áreas urbanas gracias a los cultivos en regadío entorno al río Yangtze (China); o el de Vlassova et al. (2016) que estudia el efecto del arbolado en la reducción de la temperatura en la dehesa de Extremadura (España).

En este contexto, el objetivo de este trabajo es constatar la existencia de un efecto frío en los agroecosistemas a través de la evaluación de los cambios inducidos por la implantación de cultivos de regadío en la temperatura superficial terrestre (TS). Adicionalmente, se determinan los factores que explican dichos cambios para la evaluación de usos del suelo.

Para la consecución del objetivo se estudiará el cultivo de cítricos en regadío en el Campo de Cartagena (SE - España). Esta zona de estudio es una comarca agraria del mediterráneo occidental que en los últimos 40 años ha sufrido un profundo cambio de los usos del suelo, pasando de orientaciones de secano a regadío, especialmente de cítricos (Alcon et al., 2020). Un fenómeno similar ha sucedido en otras áreas del mediterráneo español (Valera et al., 2017), de California (Roberts et al., 2015) o de Florida (Volk et al., 2017), lo que hace que el caso de estudio tenga un alcance que supera a su importancia local. 


\section{Materiales y métodos}

\section{Área de estudio}

La comarca agraria del Campo de Cartagena, ubicada en la Región de Murcia (sureste de España) (Figura N01), está afectada por un clima semiárido, con temperaturas y precipitaciones medias anuales de $18^{\circ} \mathrm{C}$ y $350 \mathrm{~mm}$ respectivamente. Enero y agosto son los meses con las temperaturas medias mínimas $\left(10,90^{\circ} \mathrm{C}\right)$ y máximas de esta zona $\left(25,97^{\circ} \mathrm{C}\right)$, mientras que en julio $(0,82 \mathrm{~mm})$ y septiembre $(56,96 \mathrm{~mm})$ tiene lugar las precipitaciones medias mínimas y máximas (Martínez-Paz et al., 2016; Perni et al., 2020). En cuanto a la vegetación predominante de la zona de estudio (libre de cultivo) destaca el palmito y el espino negro.

Figura $\mathrm{N}^{01}$

Área de estudio

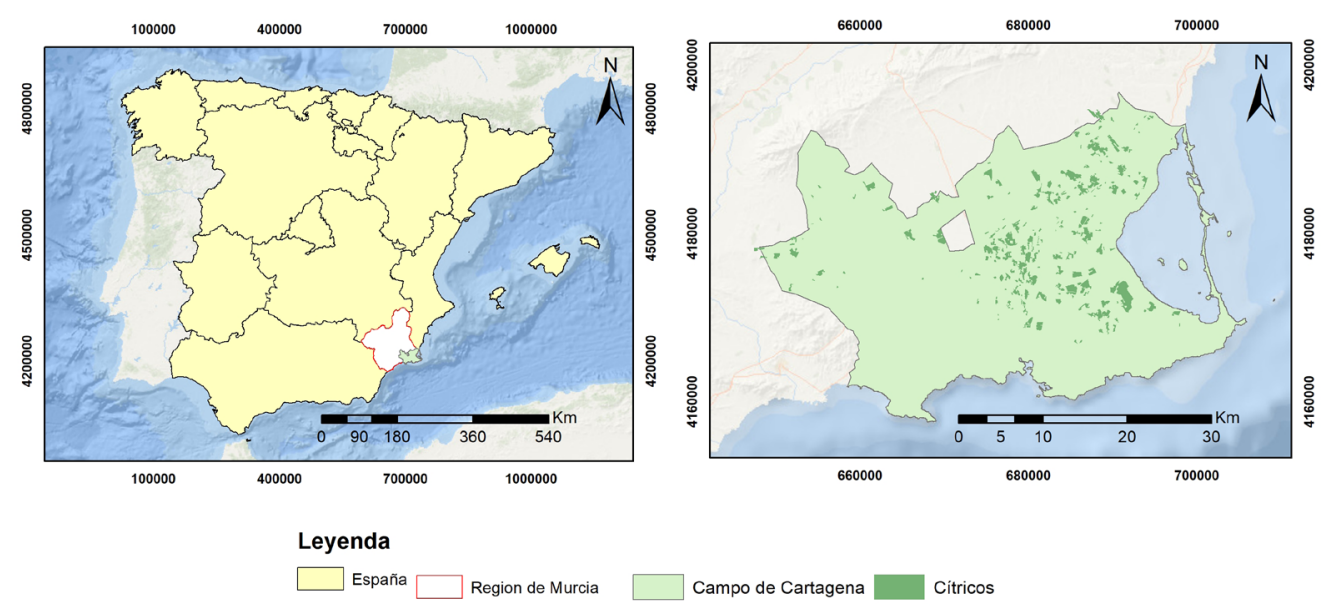

Fuente: elaboración propia.

Los aprovechamientos conjuntos de recursos hídricos procedentes del Trasvase Tajo-Segura y aguas subterráneas (Pellicer-Martínez \& Martínez-Paz, 2018a) han llevado a que esta comarca haya sufrido en las últimas décadas un cambio muy importante en los usos del suelo y en las orientaciones productivas presentes (Alcon et al., 2014). Así pues, en el año 1984, de las 58.046 ha de superficie agrícola, el $52 \%$ correspondía al regadío, mientras que, en el año 2016 , de las 61.326 ha cultivadas, el $77,34 \%$ es regadío, relegando a las tierras en secano al $22,66 \%$ restante (CREM, 2018). Dentro del regadío destaca por su extensión (8.000 ha) el cultivo de los cítricos (limonero, naranjo, mandarino y pomelo), localizados en parcelas de muy diferentes tamaños y en su mayoría próximas a la costa (Alcon et al., 2020). En cuanto al secano destaca el cultivo del almendro (4.500 ha), ubicado principalmente en la parte más occidental de la zona de estudio. La importancia de la agricultura regional queda reflejada en la aportación de este sector al PIB. En el año 2018 el VAB agrario regional representó el 5,43\% del PIB, superando el 2,69\% del promedio nacional (CREM, 2018). 


\section{Metodología}

Para el desarrollo del trabajo se ha obtenido información de las 216 parcelas de cítricos censadas en el Campo de Cartagena en el año 2016. Para cada una de ellas se ha medido tanto su TS como los distintos factores que, a priori y basándose en la revisión de los trabajos previos sobre el tema (Karnieli et al., 2010; Farg et al., 2017; Liou et al., 2017) podrían explicar su variabilidad: temperatura del aire, índice de vegetación, índice de contenido de agua de vegetación, distancia de la parcela a la costa, altitud, pendientes y orientaciones.

Las técnicas utilizadas en su obtención son la teledetección y la explotación de bases de datos espaciales mediante herramientas SIG. Para el tratamiento estadístico se utilizó el software Gretl con el que se llevó a cabo pruebas t de Student, modelos de regresión lineal múltiple y predicciones.

Para contrastar la influencia del regadío en la TS se han tomado como referencia los años 1984 (inicio del periodo de transformación en la zona de secano a regadío por la aportación de aguas del trasvase) y 2016.

La TS (en ${ }^{\circ} \mathrm{C}$ ) se ha basado en la información contenida en imágenes satélites de los sensores remotos Landsat 5-TM y Landsat 8-OLI-TIRS. Las mismas, que proceden del Servicio Geológico de los Estados Unidos (USGS), cuentan con bandas multiespectrales con una resolución espacial de $30 \mathrm{~m}$ (en bandas térmicas $120 \mathrm{~m}$ y $100 \mathrm{~m}$ para Landsat 5 y 8 respectivamente), y sistema de coordenadas ETRS89. Estas imágenes se capturaron en condiciones claras ( $0 \%$ de cobertura de nubes), en fechas de verano (el 19 de Julio de 1984 y el 27 de Julio de 2016), y han sido corregidas radiométricamente, pasando valores de Niveles Digitales a valores por encima de la atmósfera.

Para el cálculo de la TS se ha utilizado la técnica de Chamber \& Markham (2003), que es la propuesta por la USGS (2013), la cual no requiere de variables climáticas y es utilizada generalmente para obtener esta variable de la información satelital a partir de la expresión (Orimoloye et al., 2018; Carvajal \& Pabón, 2014):

$$
\mathrm{TS}=\frac{\mathrm{T}_{\mathrm{B}}}{1+\mathrm{W} *\left(\frac{T_{\mathrm{B}}}{\mathrm{P}}\right) * \ln (\mathrm{LSE})}
$$

donde: $\mathrm{T}_{\mathrm{B}}=$ temperatura de brillo, $\mathrm{W}=$ longitud de onda de radiación emitida, $\mathrm{P}=$ constante de 14.380, y LSE= factor de corrección de emisividad propuesto por Sobrino et al. (2004), siendo las dos primeras variables proporcionadas por la imagen utilizada.

Asimismo, y para explicar factores de TS se ha obtenido información de las siguientes variables:

-Índices de vegetación de diferencia normalizada (NDVI) y de contenido de agua de vegetación de diferencia normalizada (NDWI) obtenidas de las mismas imágenes satélites:

$$
\mathrm{NDVI}=\frac{\mathrm{IRC}-\mathrm{R}}{\mathrm{IRC}+\mathrm{R}}
$$


$\mathrm{NDWI}=\frac{\text { IRC-IRM }}{\text { IRC }+ \text { IRM }}$

Siendo (para Landsat 5 y 8): IRC el Infrarrojo cercano (bandas 4 y 5); R el Rojo Visible (bandas 3 y 4); e IRM el Infrarrojo medio (bandas 5 y 6 ).

Estos índices han sido seleccionados para obtener el grado de desarrollo y calidad de la vegetación, así como su contenido de agua. Los valores de estos índices oscilan entre -1 y 1. Cuanto mayor sea su valor, más saludable y densa será la vegetación (Santana et al., 2010). Para el caso del NDVI, valores próximos a 0 indican una ausencia de vegetación, mientras que por encima de este valor habrá una mayor densidad vegetal. Para el NDWI, Gao (1996) plantea un valor mínimo apropiado de 0,05; por lo tanto, un aumento de los valores de este índice indicará un nivel más alto de humedad en las cubiertas vegetales. Se espera que estos índices tengan una relación negativa con la TS del ecosistema agrario estudiado.

-Temperaturas medias del aire (AirT) en ${ }^{\circ} \mathrm{C}$, a partir de la interpolación Spline de los valores de Taire de las estaciones meteorológicas de la zona de estudio (SIAM, 2018).

-Elevación media (m), Pendiente media ( ${ }^{\circ}$ y Orientaciones (-1: umbría, 0: plano, 1: solana) procedentes del Modelo Digital de Elevaciones (MDE) (CNIG, 2018).

-Lejanía media a la costa (Lejanía costa) en $\mathrm{Km}$, de las parcelas de cítricos en regadío, obtenidas del Sistema de Información sobre Ocupación del Suelo en España (SIOSE).

A modo de resumen, en el Cuadro N01 se presenta la descriptiva de estas variables para las 216 parcelas de cítricos consideradas.

Cuadro No1

Estadísticos descriptivos de las variables

\begin{tabular}{|l|r|r|r|r|}
\hline Variables & \multicolumn{1}{|c|}{ Media } & Desv. Típica & \multicolumn{1}{c|}{ Mín } & \multicolumn{1}{c|}{ Máx } \\
\hline TS2016 $\left({ }^{\circ} \mathrm{C}\right)$ & 32,470 & 1,533 & 28,540 & 36,090 \\
\hline TS1984 $\left({ }^{\circ} \mathrm{C}\right)$ & 33,710 & 1,919 & 27,090 & 37,220 \\
\hline NDVI2016 $(-1,1)$ & 0,342 & 0,137 & 0,082 & 0,668 \\
\hline NDWI2016 $(-1,1)$ & 0,065 & 0,088 & $-0,095$ & 0,313 \\
\hline Lejanía costa $(\mathrm{Km})$ & 9,647 & 6,089 & 0,504 & 31,090 \\
\hline Elevación $(\mathrm{m})$ & 58,560 & 52,060 & 6,000 & 282,000 \\
\hline Pendientes $\left({ }^{\circ}\right)$ & 1,497 & 2,311 & 0,000 & 12,140 \\
\hline AirT2016 $\left({ }^{\circ} \mathrm{C}\right)$ & 25,900 & 2,592 & 24,350 & 27,070 \\
\hline AirT1984 $\left({ }^{\circ} \mathrm{C}\right)$ & 24,990 & 0,990 & 22,240 & 26,280 \\
\hline Orientaciones $(\%)$ & -1 (umbría): 7,43 & & & \\
\hline & 0 (Plano): 75,25 & & & \\
\hline & 1 (Solana): 17,32 & & & \\
\hline
\end{tabular}

Fuente: elaboración propia. 


\section{Resultados y discusión}

La distribución espacial de TS y los usos del suelo para los dos años objeto de estudio en el Campo de Cartagena viene recogida en las Figuras $N^{\circ} 2$ y 3 . En estas Figuras, para la elaboración del mapa de usos del suelo se utilizó el Corine Land Cover 1990-2012, el SIOSE, y validación de estas capas mediante visualización de ortofotos y aplicación del índice NDVI para dos imágenes satélite (2016 y 1984).

En ambos años la TS es más alta en el oeste y más baja en el este y sur, mostrando una tendencia general al alza del sureste al noroeste. En el año 2016, las TS localizadas al este de la comarca agraria son mucho más bajas, correspondiente a la agricultura de regadío de la zona, siendo más altas al oeste, donde hay una menor extensión del regadío. En cambio, en el año 1984, no se localizan extensas áreas de bajas temperaturas asociadas al regadío. Además, la TS de las parcelas de cítricos muestra diferencias temporales, siendo más alta en $1984\left(33,71^{\circ} \mathrm{C}\right)$ que en $2016\left(32,47^{\circ} \mathrm{C}\right)$.

Figura $\mathrm{N}^{0} 2$

TS $\left({ }^{\circ} \mathrm{C}\right)(\mathrm{a})$ y usos del suelo (b) en el año 1984 del área de estudio

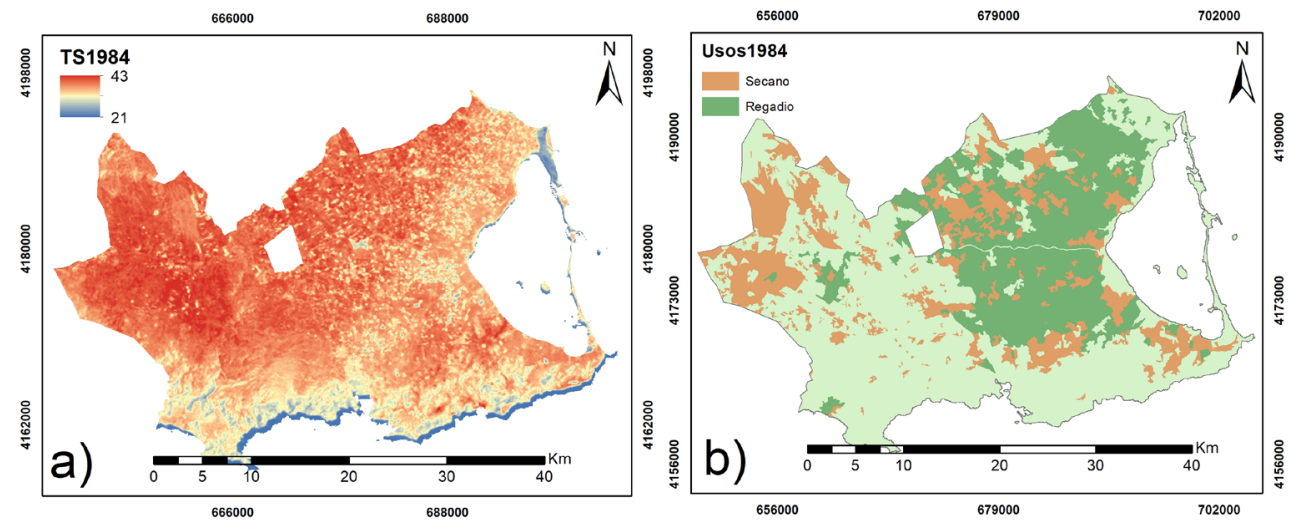

Fuente: elaboración propia.

Figura $\mathrm{N}^{\circ} 3$

TS $\left({ }^{\circ} \mathrm{C}\right)(a)$ y usos del suelo $(b)$ en el año 2016 del área de estudio
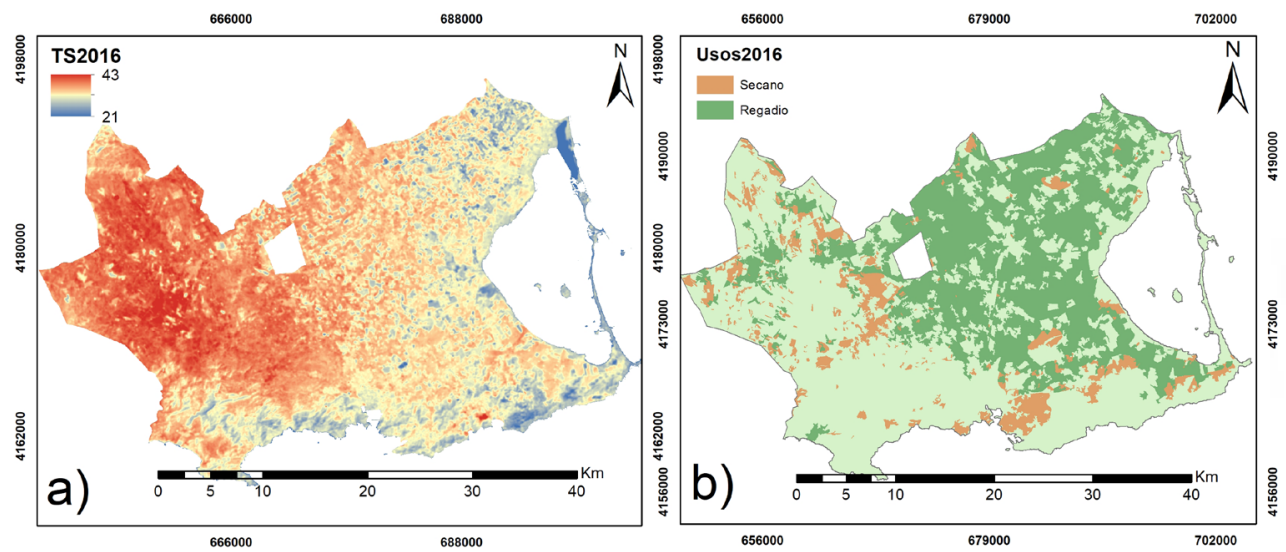

Fuente: elaboración propia. 


\section{Análisis de las diferencias de TS}

Las diferencias en TS que puedan estar asociadas al efecto frío del regadío han sido analizadas para los años de estudio, la temperatura del aire y el uso del suelo a través de un contraste de diferencia de medias. El contraste de las diferencias temporales de TS sobre las parcelas de cítricos censadas en 2016 indican que dichas diferencias son significativas (sig. <0.01), con unas temperaturas medias en superficie mayores $\left(2,47^{\circ} \mathrm{C}\right)$ en el año 1984 que en el 2016.

Dado que la diferencia temporal de TS puede venir explicada por la diferente temperatura del aire (AirT) en los días en los que se realizó la medida, se ha llevado a cabo un contraste de diferencia de temperaturas del aire. Esto confirmó que existe una diferencia significativa de casi $1^{\circ}$ $\mathrm{C}\left(0,93^{\circ} \mathrm{C}\right)$ en AirT entre ambos años. Pero es en el año 1984, el que tiene una TS más alta, el que registra una AirT más baja, de modo que esta no es la razón de las diferencias de TS interanuales encontradas.

Por otro lado, para comprobar si el distinto uso de suelo puede ser el responsable de las diferencias en TS, la comparación de la TS de las parcelas en 1984 ha sido realizada distinguiendo entre secano y regadío en dicha fecha. Para ello, y haciendo uso de la ortofoto del Plan Nacional de Observación del Territorio (PNOA) en dicho año junto con la capa Corine Land Cover, se calculó el índice NDVI de las parcelas para la imagen satélite del año 1984 con la finalidad de distinguir las áreas de secano de las de regadío (NDVI> 0,2). Con este análisis se vio que existía una distribución igualitaria del tipo de uso en las parcelas ( $51 \%$ de regadío y un $49 \%$ de secano) en el año 1984 . El test de diferencia de medias de las TS de las dos muestras (Regadío1984 vs Secano1984) revela la existencia de diferencias estadísticamente significativas, siendo la TS media de las parcelas de secano $2,09^{\circ} \mathrm{C}$ superior a las del regadío. Este resultado confirma que las diferencias de TS vienen explicadas por el distinto uso del suelo, con un considerable efecto frío del regadío del orden del $8,2 \%$ de la TS del secano.

\section{Factores explicativos de la TS}

Una vez comprobado el efecto frío del regadío, dada su capacidad para reducir la temperatura en superficie (TS) se analizó, para el año 2016, los factores explicativos de dicha TS para los cítricos de regadío del Campo de Cartagena. Para ello se ha estimado un modelo de regresión lineal múltiple (Cuadro $N^{\circ} 2$ ) en el que se ha considerado 7 factores que pueden tener efecto sobre dicha temperatura, y cuya estadística descriptiva se presentó en el Cuadro $\mathrm{N} N 1$.

En primer lugar, se ha realizado la estimación de un modelo general con todas las variables (Estimación inicial). Sobre este modelo se han eliminado de manera individualizada las variables no significativas según el criterio de la menor significación (mayor p-valor) para alcanzar un modelo anidado más parsimonioso (Estimación final), donde las variables explicativas poseen un nivel de significación mínimo del 99\%. Del análisis de la estimación final se aprecia que los factores que se relacionan con la TS en regadío son el NDVI, el NDWI y la lejanía de la parcela a la costa. Estos tres factores logran explicar el $72 \%$ de la varianza de la TS, siendo un ajuste notable dado que el modelo no presenta problemas de multicolinealidad o heterocedasticidad (Greene, 1997). Se han ensayado otras especificaciones no lineales (cuadráticas y con interacciones entre las variables independientes) que no han mejorado en ningún caso el ajuste de los datos. 
Así, dado el signo de los coeficientes del modelo, un mayor índice de vegetación (NDVI) y de contenido de agua de vegetación (NDWI) o una menor lejanía a la costa reducen la temperatura en superficie de la parcela. Atendiendo al valor de dichos coeficientes, y dado el carácter lineal, cada punto adicional del índice de vegetación (NVDI) y de contenido de agua de la vegetación (NDWI) reducen la TS en 2,88 y $7,01^{\circ} \mathrm{C}$ respectivamente, mientras que cada kilómetro de distancia al mar de la parcela aumenta la $\mathrm{TS}$ en $0,11^{\circ} \mathrm{C}$.

\section{Cuadro $\mathrm{N}^{\circ} 2$}

Modelos de TS $\left({ }^{\circ} \mathrm{C}\right)$

\begin{tabular}{|l|r|r|}
\hline Variables & Estimación inicial & Estimación final \\
\hline Constante & $33,001(3,951)^{\star * *}$ & $32,824(0,260)^{\star * *}$ \\
\hline NDVI2016 & $-2,786(0,865)^{\star * *}$ & $-2,881(0,861)^{\star * *}$ \\
\hline NDWI2016 & $-7,268(1.337)^{* * *}$ & $-7,010(1,331)^{\star * \star}$ \\
\hline Lejanía costa & $0,099(0.021)^{\star * *}$ & $0,113(0,010)^{\star * *}$ \\
\hline Elevación & $1,141(0,002)$ & - \\
\hline Pendientes & $0,0003(0,0001)$ & - \\
\hline AirT2O16 & $-0,004(0,149)$ & - \\
\hline Orientaciones & $-0,155(0,107)$ & 0,722 \\
\hline$R^{2}$ & 0,714 & $186,790(0,000)$ \\
\hline Estadístico F & $69,447(0,000)$ & 216 \\
\hline$N^{\circ}$ observaciones & 216 & - \\
\hline
\end{tabular}

Variable dependiente: TS. Los errores estándar se presentan entre paréntesis. ${ }^{* *}$ indican Valor $\mathrm{P}$ a niveles de significación de 0,01

Fuente: elaboración propia.

La relación inversa de la TS y el NDVI mostrada en este estudio se encuentra acorde con los resultados de trabajos como los de Sun \& Chen (2017) y Ferrelli et al. (2018), confirmando que la vegetación reduce las temperaturas en superficie mediante la transferencia de calor latente de la superficie a la atmósfera a través de la evapotranspiración.

Asimismo, los valores de TS exhibieron una relación inversa con el contenido de agua de la vegetación (NDWI) como muestran, a su vez, los trabajos de Farg et al. (2017) y Jiang et al. (2015), demostrando la importancia del agua en los cultivos para la reducción de temperaturas en superficie.

En cuanto a la lejanía de la costa, se confirmó la influencia que tiene el Mar Menor sobre la reducción de las TS de la comarca agraria. Este resultado ha sido contrastado en el estudio de la isla de calor urbana, comprobándose como los cuerpos de agua reducen las temperaturas en superficie (Acero et al., 2013; Moyer \& Hawkins, 2017; Cai et al., 2018). Sin embargo, apenas hay trabajos que hayan considerado esta variable en el caso de ecosistemas agrarios (Cheval \& Constantin, 2018).

\section{Predicciones de cambios de uso del suelo}

Además de la identificación de los factores que explican la TS del sistema agrario estudiado, el modelo estimado tiene una utilidad predictiva. Así, se puede evaluar los cambios de TS debido 
a una posible disminución de los caudales para riego disponibles en la zona (Pellicer-Martínez \& Martínez-Paz, 2018b) que daría lugar a la sustitución de parcelas de riego por otras de cultivos en secano.

Este cambio de orientación productiva modificaría el valor de los índices espectrales (NDVI y NDWI), que tomarían el valor de los cultivos de secano que se implantarán, permaneciendo invariante el de la lejanía de las parcelas al mar. Así, de la estimación de los valores de ambos índices para los cultivos de secano presentes actualmente en la zona (almendro, cereales para grano y pastizales), ha sido posible predecir la TS para las 216 parcelas en riego si estas fueran ocupadas por cada uno de dichos cultivos. Estos resultados se muestran en el Cuadro № 3, donde también se presenta el valor predicho por el modelo para los cítricos en regadío.

Cuadro $\mathrm{N}^{\circ} 3$

Predicción media de TS ( $\left.{ }^{\circ} \mathrm{C}\right)$ según tipo de cultivos

\begin{tabular}{|l|r|r|r|r|}
\hline \multicolumn{1}{|c|}{ Cultivos } & NDVI $(-1,1)$ & NDWI $(-1,1)$ & \multicolumn{1}{c|}{ TS predicha } & \multicolumn{1}{c|}{ Intervalo 95\% } \\
\hline Almendros & 0,180 & $-0,033$ & 33,598 & $32,033-35,164$ \\
\hline Herbáceos & 0,169 & $-0,039$ & 33,673 & $32,107-35,240$ \\
\hline Pastizales & 0,187 & $-0,044$ & 33,651 & $32,086-35,217$ \\
\hline Cítricos & 0,342 & 0,065 & 32,442 & $30,882-34,002$ \\
\hline
\end{tabular}

Fuente: elaboración propia.

Al comparar la TS predicha para los cultivos de secano alternativos con los valores predichos para los cítricos de regadío se aprecia una TS media significativamente más alta en los tres cultivos de secano respecto a los cítricos de regadío, con diferencias de hasta $1,23^{\circ} \mathrm{C}$ (Cuadro $\mathrm{N}^{\circ} 4$ ).

Cuadro $\mathrm{N}^{\circ} 4$

Contraste de diferencias de medias de temperatura por tipo de cultivos

\begin{tabular}{|l|l|r|r|r|}
\hline \multicolumn{2}{|c|}{ Variables } & t-Test & P-valor & Diferencia Media \\
\hline Almendros & Cítricos & Pareado & 0,000 & 1,16 \\
\hline Herbáceos & Cítricos & Pareado & 0,000 & 1,23 \\
\hline Pastizales & Cítricos & Pareado & 0,000 & 1,21 \\
\hline
\end{tabular}

Fuente: elaboración propia.

De este modo, es posible considerar los efectos que pueden tener, sobre las TS de esta comarca, las futuras planificaciones de regadíos destinadas a reducir la contaminación difusa (PeIlicer-Martínez \& Martínez-Paz, 2016) o las políticas que restrinjan la disponibilidad de agua en la zona (Perni \& Martínez-Paz, 2013), las cuales transformarían las actuales parcelas de cítricos en secanos, alterando el fenómeno efecto frío, y, por tanto, la oferta de SE de regulación climática.

Aunque este cambio en las temperaturas superficiales puede parecer pequeño, tiene gran importancia dada su influencia en la actividad y diversidad de los organismos del suelo (EC, 2010), en la avifauna (Böhning-Gaese et al., 2008) y en la biodiversidad en general (Scarano, 2019). Por lo tanto, los servicios ecosistémicos de regulación, como el aquí estudiado, juegan un papel crucial en el conjunto de servicios ecosistémicos proporcionados por los agroecosistemas (Blanco-Can- 
qui et al., 2015). Además, en ecosistemas agrarios es bien sabido que la provisión de sombra y reducción de temperaturas permite aumentar la infiltración y retención del agua, regular la calidad del aire, y reducir la emisión de gases de efecto invernadero (Smith et al., 2013).

\section{Conclusiones}

Este trabajo ha presentado un método para evaluar la provisión de servicios ecosistémicos de regulación climática, en concreto la reducción de la temperatura de la superficie terrestre (TS), por un agroecosistema de regadío. Para la citricultura del Campo de Cartagena, el mismo se ha cuantificado en una reducción de entre 1,16 y $1,23^{\circ} \mathrm{C}$ de la TS, la cual viene explicada básicamente por los mayores índices de vegetación e índices de contenido en agua de estos cultivos.

Este artículo tiene sus limitaciones. La TS está cambiando todo el tiempo y sólo se utilizó una imagen satélite para los años 2016 y 1984. Estudios anteriores (Kang \& Eltahir, 2017) mostraron diferencias en la TS entre días y estaciones, pero algunos factores, como el NDVI, pueden no tener efecto en la TS durante la noche o el invierno. Por lo tanto, los resultados de este análisis espacial deben ser complementados por estudios adicionales que analicen el efecto multitemporal del efecto frío del regadío. Además, se recomendaría una comparación espacial entre diferentes zonas agrícolas en diferentes condiciones climáticas.

Estos resultados pueden ser generalizados a otras comarcas agrarias dedicadas a la citricultura, como las encontradas en el levante español u otras zonas de producción citrícola en climas similares. Constituye, por tanto, una herramienta apropiada para completar la evaluación de los servicios ecosistémicos de los agroecosistemas, dado que permite cuantificar uno de los indicadores relevantes de los SE de regulación climática.

\section{Agradecimientos}

Este trabajo se ha realizado en el marco de los proyectos AGRISERVI (AGR2015-64411-R), financiado por el Ministerio de Economía y Competitividad (MINECO), y el Proyecto 20912/PI/18 financiado por "Fundación Séneca-Agencia de Ciencia y Tecnología de la Región de Murcia". Forma parte de la investigación predoctoral del primer autor el trabajo, financiada por el Ministerio de Educación, Cultura y Deporte (MECD) (FPU16/03562).

\section{Referencias}

ACERO, J.A.; ARRIZABALAGA, J.; KUPSKI, S. \& KATZSCHNER, L. Urban heat island in a coastal urban area in northern Spain. Theoretical and Applied Climatology, 2013, Vol. 113, № 1-2, p. 137-154. https://doi.org/10.1007/s00704-012-0774-z

ALBALADEJO-GARCÍA, J.A.; ALCON, F.; MARTÍNEZ-PAZ, J.M. Efecto del regadío sobre la reducción de la temperatura de la superficie terrestre. El caso de los cítricos del Campo de Cartagena, Re- 
gión de Murcia. En: UNIVERSIDAD DE MURCIA (eds.). IV Jornadas Doctorales de la Universidad de Murcia, Murcia, 2019, p. 727-731.

ALCON, F.; TAPSUWAN, S.; MARTÍNEZ-PAZ, J.M.; BROUWER, R. \& DE MIGUEL, M.D. Forecasting deficit irrigation adoption using a mixed stakeholder assessment methodology. Technological Forecasting and Social Change, 2014, Vol. 83, p. 183-193. https://doi.org/10.1016/j.techfore.2013.07.003

ALCON, F.; DE MIGUEL, M.D. \& MARTÍNEZ-PAZ, J.M. Assessment of real and perceived cost-effectiveness to inform agricultural diffuse pollution mitigation policies. Land Use Policy, 2020, Vol. 97, 104561. https://doi.org/10.1016/j.landusepol.2020.104561

BLANCO-CANQUI, H.; SHAVER, T.M.; LINDQUIST, J.L.; SHAPIRO, C.A.; ELMORE, R. W.; FRANCIS, C. A. \& HERGERT, G. W. Cover crops and ecosystem services: Insights from studies in temperate soils. Agronomy Journal, 2015, Vol. 107, № 6, p. 2449-2474. https://doi.org/10.2134/agronj15.0086

BÖHNING-GAESE, K.; JETZ, W. \& SCHAEFER, H.C. Impact of climate change on migratory birds: community reassembly versus. Global Ecology and Biogeography, 2008, Vol. 17, No 1, p. 38-49. https://doi.org/10.1111/j.1466-8238.2007.00341.x

CAI, Z.; HAN, G. \& CHEN, M. Do water bodies play an important role in the relationship between urban form and land surface temperature? Sustainable Cities and Society, 2018, Vol. 39, p. 487498. https://doi.org/10.1016/j.scs.2018.02.033

CARVAJAL, A.F. \& PABÓN, J.D. Temperatura de la superficie terrestre en diferentes tipos de cobertura de la región andina colombiana. Sociedade \& Natureza, 2014, Vol. 26, No 1, p. 95-112. https:// doi.org/10.1590/1982-451320140107

CHANDER, G. \& MARKHAM, B. Revised Landsat-5 TM radiometric calibration procedures and postcalibration dynamic ranges. IEEE Transactions on geoscience and remote sensing, 2003, Vol. 41, No 11, p. 2674-2677. https://doi.org/10.1109/TGRS.2003.818464

CHEN, F.; XU, X.; BARLAGE, M.; RASMUSSEN, R.; SHEN, S.; MIAO, S. \& ZHOU, G. Memory of irrigation effects on hydroclimate and its modeling challenge. Environmental Research Letters, 2018, Vol. 13, No 6, p. 064009. https://doi.org/10.1088/1748-9326/aab9df

CHEVAL, S. \& CONSTANTIN, S. Black Sea impact on its west-coast land surface temperature. Theoretical and Applied Climatology, 2018, p. 1-11. https://doi.org/10.1007/s00704-018-2454-0

CNIG (Centro Nacional de Información Geográfica). Modelos Digitales de Elevaciones y SIOSE, 2018. (Consulta: 12/05/2020). http://centrodedescargas.cnig.es/CentroDescargas/index.jsp

COOK, B.I.; PUMA, M.J. \& KRAKAUER, N.Y. Irrigation induced surface cooling in the context of modern and increased greenhouse gas forcing. Climate Dynamics, 2011, Vol. 37, No 7-8, p. 1587-1600. https://doi.org/10.1007/s00382-010-0932-x 
CREM (Centro Regional de Estadística de Murcia). Censo agrario, 2018. (Consulta: 12/12/2018). http://econet.carm.es/web/crem/inicio//crem/sicrem/PU590/Indice1.html

DUVEILLER, G., HOOKER, J., \& CESCATTI, A. The mark of vegetation change on Earth's surface energy balance. Nature Communications, 2018, Vol. 9, No 1, p 679. https://doi.org/10.1038/s41467017-02810-8

EC. Soil biodiversity: functions, threats and tools for policy makers. DG Environment, European Communities, 2010, p. 254. (Consulta: 10/04/2020). http://ec.europa.eu/environment/archives/ soil/pdf/biodiversity_report.pdf

ESTOQUE, R. C., MURAYAMA, Y., \& MYINT, S. W. Effects of landscape composition and pattern on land surface temperature: An urban heat island study in the megacities of Southeast Asia. Science of the Total Environment, 2017, Vol. 577, p. 349-359. https://doi.org/10.1016/j.scitotenv.2016.10.195

FARG, E.; ARAFAT, S.; EL-WAHED, M.A. \& EL-GINDY, A. Evaluation of water distribution under pivot irrigation systems using remote sensing imagery in eastern Nile delta. The Egyptian Journal of Remote Sensing and Space Science, 2017, Vol. 20, p. 13-19. https://doi.org/10.1016/j.ejrs.2016.12.001

FERRELLI, F.; CISNEROS, M.A.H.; DELGADO, A.L. \& PICCOLO, M.C. Spatial and temporal analysis of the LST-NDVI relationship for the study of land cover changes and their contribution to urban planning in Monte Hermoso, Argentina. Documents d'anàlisi geográfica, 2018, Vol. 64, No 1, p. 25 47. https://doi.org/10.5565/rev/dag.355

GUIMBERTEAU, M.; LAVAL, K.; PERRIER, A. \& POLCHER, J. Global effect of irrigation and its impact on the onset of the Indian summer monsoon. Climate Dynamics, 2012, Vol. 39, No 6, p. 1329-1348. https://doi.org/10.1007/s00382-011-1252-5

GREENE, W.H. Econometric Analysis. New York: Editorial Pearson, 1997.

HAINES-YOUNG, R. \& POTSCHIN, M. Common international classification of ecosystem services (CICES, Version 4.1). European Environment Agency, 2012, Vol. 33.

HEREHER, M.E. Effects of land use/cover change on regional land surface temperatures: severe warming from drying Toshka lakes, the Western Desert of Egypt. Natural Hazards, 2017, Vol. 88, No 3, p. 1789-1803. https://doi.org/10.1007/s11069-017-2946-8

JIANG, Y.; FU, P. \& WENG, Q. Assessing the impacts of urbanization-associated land use/cover change on land surface temperature and surface moisture: a case study in the Midwestern United States. Remote Sensing, 2015, Vol. 7, No 4, p. 4880-4898. https://doi.org/10.3390/rs70404880

KANG, S., \& ELTAHIR, E. A. Impact of irrigation on regional climate over Eastern China. Geophysical Research Letters, 2019, Vol. 46, No 10, p. 5499-5505. https://doi.org/10.1029/2019GL082396 
KARNIELI, A.; AGAM, N.; PINKER, R.T.; ANDERSON, M.; IMHOFF, M.L.; GUTMAN, G.G.; PANOV, N. \& GOLDBERG, A. Use of NDVI and land surface temperature for drought assessment: Merits and limitations. Journal of Climate, 2010, Vol. 23, No 3, p. 618-633. https://doi.org/10.1175/2009JCLI2900.1

LI, W., CAO, Q., LANG, K., \& WU, J. Linking potential heat source and sink to urban heat island: Heterogeneous effects of landscape pattern on land surface temperature. Science of the Total Environment, 2017, Vol. 586, p. 457-465. https://doi.org/10.1016/j.scitotenv.2017.01.191

LIOU, Y.A.; NGUYEN, A.K. \& LI, M.H. Assessing spatiotemporal eco-environmental vulnerability by Landsat data. Ecological Indicators, 2017, Vol. 80, p. 52-65. https://doi.org/10.1016/j.ecolind.2017.04.055

LOBELL, D.B.; BONFILS, C.J.; KUEPPERS, L.M. \& SNYDER, M.A. Irrigation cooling effect on temperature and heat index extremes. Geophysical Research Letters, 2008, Vol. 35, No 9, p. 1-5. https:// doi.org/10.1029/2008GL034145

MARTÍNEZ-PAZ, J.M.; PERNI, A.; RUIZ, P. \& PELLICER, F. Valoración económica de los fallos de suministro en los regadíos de la cuenca del Segura. Revista Española de Estudios Agrosociales y Pesqueros, 2016, Vol. 244, p. 35-67.

MEA (Millennium Ecosystem Assessment). Ecosystems and human well-being: policy responses: findings of the responses working group of the millennium ecosystem assessment. Millennium Ecosystem Assesment, 2005.

MOYER, A.N. \& HAWKINS, T.W. River effects on the heat island of a small urban area. Urban Climate, 2017, Vol. 21, p. 262-277.https://doi.org/10.1016/j.uclim.2017.07.004

ORIMOLOYE, I.R.; MAZINYO, S.P.; NEL, W. \& KALUMBA, A.M. Spatiotemporal monitoring of land surface temperature and estimated radiation using remote sensing: human health implications for East London, South Africa. Environmental Earth Sciences, 2018, Vol. 77, No 3, p. 77. https://doi. org/10.1007/s12665-018-7252-6

PELLICER-MARTÍNEZ, F. \& MARTÍNEZ-PAZ, J.M. Grey water footprint assessment at the river basin level: Accounting method and case study in the Segura River Basin, Spain. Ecological Indicators, 2016, Vol. 60, p. 1173-1183. https://doi.org/10.1016/j.ecolind.2015.08.032

PELLICER-MARTÍNEZ., F. \& MARTÍNEZ-PAZ, J.M. Probabilistic evaluation of the water footprint of a river basin: Accounting method and case study in the Segura River Basin, Spain. Science of the Total Environment, 2018a, Vol. 627, p. 28-38. https://doi.org/10.1016/j.scitotenv.2018.01.223

PELLICER-MARTÍNEZ, F. \& MARTÍNEZ-PAZ, J.M. Climate change effects on the hydrology of the headwaters of the Tagus River: implications for the management of the Tagus-Segura transfer. Hydrology and Earth System Sciences, 2018b, Vol. 22. https://doi.org/10.5194/hess-22-6473-2018 
PERNI, A., BARREIRO-HURLE, J. \& MARTÍNEZ-PAZ, J.M. When policy implementation failures affect public preferences for environmental goods: Implications for economic analysis in the European water policy. Ecological Economics, 2020, Vol. 169, 106523. https://doi.org/10.1016/j.ecolecon.2019.106523

PERNI, A. \& MARTÍNEZ-PAZ, J.M. A participatory approach for selecting cost-effective measures in the WFD context: The Mar Menor (SE Spain). The Science of the Total Environment, 2013, Vol. 458, p. 303-311. https://doi.org/10.1016/j.scitotenv.2013.04.029

ROBERTS, D.A.; DENNISON P.E.; ROTH, K.L.; DUDLEY, K. \& HULLEY, G. Relationships between dominant plant species, fractional cover and land surface temperature in a Mediterranean ecosystem. Remote Sensing of Environment, 2015, Vol. 167, p. 152-167. https://doi.org/10.1016/j. rse.2015.01.026

SÁNCHEZ-DÍAZ, B. Teledetection in ecological research as support for the conservation of biodiversity: a review. Revista Científica, 2018, Vol. 33, p. 243-253. https://doi.org/10.14483/23448350.13370

SANTANA, L.M.; ESCOBAR, L.A. \& CAPOTE, P.A. Estimación de un índice de calidad ambiental urbano, a partir de imágenes de satélite. Revista de Geografía Norte Grande, 2010, Vol. 45, p. 77-95. http://dx.doi.org/10.4067/S0718-34022010000100006

SCARANO, F.R. Biodiversity Sector: Risks of Temperature Increase to Biodiversity and Ecosystems. En: NOBRE, C.; MARENGO, J. \& SOARES, W (eds.). Climate Change Risks in Brazil, Springer, Cham, 2019, p. 131-141.

SIAM (Sistema de Información Agrario de Murcia). Informe Agrometeorológico, 2018. (Consulta: 07/04/2020). http://siam.imida.es/apex/f?p=101:1:1753201960247786

SMITH, P.; ASHMORE, M.R.; BLACK, H.I.; BURGESS, P.J.; EVANS, C.D.; QUINE, T.A.; THOMSON, A.M.; HICKS, K. \& ORR, H.G. The role of ecosystems and their management in regulating climate, and soil, water and air quality. Journal of Applied Ecology, 2013, Vol. 50, No 4, p. 812-829. https://doi. org/10.1111/1365-2664.12016

SOBRINO, J.A.; JIMÉNEZ-MUÑOZ, J.C. \& PAOLINI, L. Land surface temperature retrieval from LANDSAT TM 5. Remote Sensing of Environment, 2004, Vol. 90, No 4, p. 434-440. https://doi. org/10.1016/j.rse.2004.02.003

SUN, R. \& CHEN, L. Effects of green space dynamics on urban heat islands: Mitigation and diversification. Ecosystem Services, 2017, Vol. 23, p. 38-46. https://doi.org/10.1016/j.ecoser.2016.11.011

THIERY, W.; DAVIN, E.L.; LAWRENCE, D.M.; HIRSCH, A.L.; HAUSER, M. \& SENEVIRATNE, S.I. Present day irrigation mitigates heat extremes. Journal of Geophysical Research: Atmospheres, 2017, Vol. 122, No 3, p. 1403-1422. https://doi.org/10.1002/2016JD025740

THIERY, W., VISSER, A. J., FISCHER, E. M., HAUSER, M., HIRSCH, A. L., LAWRENCE, D. M., \& SENEVIRATNE, S. I. Warming of hot extremes alleviated by expanding irrigation. Local Cooling and Warming, 2020, Vol. 11, No 1, p. 1-7. https://doi.org/10.1038/s41467-019-14075-4 
USGS. Landsat 8 Conversion to Radiance, Reflectance and At-Satellite Brightness Temperature Using the USGS Landsat 8 Product, 2013.

VALERA, A.L.; AÑÓ, C.V. \& SÁNCHEZ, J.D. Transformación de usos agrícolas tradicionales en superficies construidas. Cambios en los usos y coberturas del suelo en el municipio de Valencia (1956-2012). Estudios Geográficos, 2017, Vol. 77, No 281, p. 671-692. http://dx.doi.org/10.3989/estgeogr.201623

VLASSOVA, L.; TUFIÑO, P.R. \& LLOVERÍA, R.M. Variabilidad espacio-temporal de la temperatura de superficie en ecosistemas de dehesa estimada mediante imágenes Landsat TM: el papel del arbolado. Geographicalia, 2016, Vol. 68, p. 69-86.

VOLK, M.I.; HOCTOR, T.S.; NETTLES, B.B.; HILSENBECK, R.; PUTZ, F.E. \& OETTING, J. Florida Land Use and Land Cover Change in the Past 100 Years. En: CHASSIGNET, E.P.; JONES, J.W.; MISRA, V. \& OBEYSEKERA, J (eds.) Florida's Climate: Changes, Variations, \& Impacts, (Edition 1). Florida, 2017, p. 51-82.

WANG, X.; GUO, W.; QIU, B.; LIU, Y.; SUN, J. \& DING, A. Quantifying the contribution of land use change to surface temperature in the lower reaches of the Yangtze River. Atmospheric Chemistry and Physics, 2017, Vol. 17, No 8, p. 4989-4996. https://doi.org/10.5194/acp-17-4989-2017

XU, L.; SHI, Z.; WANG, Y.; CHU, X.; YU, P.; XIONG, W.; ZUO, H. \& ZHANG, S. Agricultural irrigation induced climatic effects: a case study in the middle and southern Loess Plateau area, China. International Journal of Climatology, 2017, Vol. 37, N 5, p. 2620-2632. https://doi.org/10.1002/joc.4869

YUAN, X., WANG, W., CUI, J., MENG, F., KURBAN, A., \& DE MAEYER, P. Vegetation changes and land surface feedbacks drive shifts in local temperatures over Central Asia. Scientific Reports, 2017, Vol. 7, No 1, p. 1-8. https://doi.org/10.1038/s41598-017-03432-2

ZARDO, L., GENELETTI, D., PÉREZ-SOBA, M., \& VAN EUPEN, M. Estimating the cooling capacity of green infrastructures to support urban planning. Ecosystem Services, 2017, Vol. 26, p. 225-235. https://doi.org/10.1016/j.ecoser.2017.06.016 
